\title{
Influência da temperatura de rotaevaporação e do tipo de resíduo na extração de compostos bioativos de jamelão (Syzygium cumini)
}

\author{
Influence of rota-evaporation temperature and type of residue on the extraction of bioactive \\ compounds from jamelão (Syzygium cumini) \\ Influência de lá temperatura de rotaevaporizacion y ela tipo de resíduo em lá extraccion de \\ compuestos bioactivos de jamelão (Syzygium cumini)
}

Recebido: 22/01/2021 | Revisado: 25/01/2021 | Aceito: 02/02/2021 | Publicado: 09/02/2021

\author{
Bruna Stephanny Neves Brito \\ ORCID: https://orcid.org/0000-0003-4437-9932 \\ Universidade Federal de Sergipe, Brasil \\ E-mail: bruna.engdealimentos@gmail.com \\ Matheus Péricles Silva Láscaris \\ ORCID: https://orcid.org/0000-0003-4071-5716 \\ Universidade Federal de Sergipe, Brasil \\ E-mail: matheus1709@hotmail.com \\ Jane de Jesus da Silveira Moreira \\ ORCID: https://orcid.org/0000-0001-7586-4856 \\ Universidade Federal de Sergipe, Brasil \\ E-mail: jjsm.ufs@gmail.com \\ Tatiana Pacheco Nunes \\ ORCID: https://orcid.org/0000-0001-9106-8622 \\ Universidade Federal de Sergipe, Brasil \\ E-mail: tpnunes@uol.com.br \\ Alessandra Almeida Castro Pagani \\ ORCID: https://orcid.org/0000-0003-4191-3888 \\ Universidade Federal de Sergipe, Brasil \\ E-mail: alespagani@yahoo.com.br \\ Gabriel Francisco da Silva \\ ORCID: https://orcid.org/0000-0002-9622-2518 \\ Universidade Federal de Sergipe, Brasil \\ E-mail: gabriel@ufs.br
}

\begin{abstract}
Resumo
O jamelão é um fruto rico em compostos bioativos, entretanto altamente perecível e pouco utilizado pela indústria. Visto que não existe literatura sobre o efeito da temperatura de rotaevaporação e do tipo de resíduo na extração de compostos bioativos, o objetivo deste trabalho foi verificar a influência desses fatores na extração destes compostos, e consequentemente nas atividades antioxidante e antimicrobiana. Parte do resíduo foi obtida mecanicamente (bagaço), e a outra parte manualmente (casca). O bagaço e a casca foram desidratados a $40^{\circ} \mathrm{C}$ e os extratos foram preparados utilizando uma proporção de 1:10 (m/v) de álcool etílico 70\%. Após a homogeneização, os extratos foram filtrados a vácuo e rotaevaporados a $40^{\circ} \mathrm{C}$ e $50^{\circ} \mathrm{C}$. Foi possível verificar que a extração do teor de compostos fenólicos totais, flavonoides e antocianinas foi significativamente maior a $50^{\circ} \mathrm{C}$. Em relação à comparação entre os tipos de resíduos, a casca apresentou-se como melhor fonte extratora para os teores de flavonoides, antocianina, atividade antioxidante (DPPH e FRAP) e atividade antimicrobiana, embora para os compostos fenólicos totais não foi observada diferença significativa entre os tipos de resíduos. Quanto à capacidade antimicrobiana, observou-se que os extratos foram efetivos frente a Listeria monocytogenes, Bacilus cereus e Staphylococcus aureus, sendo o da casca o mais eficiente. Assim, os extratos da casca produzidos a $50^{\circ} \mathrm{C}$ podem ser considerado uma fonte potencial de estudos para trazer benefícios nutricionais, como também para a redução de resíduos no impacto ambiental, podendo ser acrescentado em novos produtos pela indústria alimentícia, farmacêutica e afins.
\end{abstract}

Palavras-chave: Casca; Bagaço; Atividade antioxidante; Atividade antimicrobiana.

\begin{abstract}
Jamelão is a fruit rich in bioactive compounds, however highly perishable and little used by the industry. Since there is not literature on the effect of rota-evaporation temperature and type of residue on the extraction of bioactive compounds, the aim of this study was to verify the influence of these factors on the extraction of these bioactive compounds, and consequently on antioxidant and antimicrobial activities. Part of the residue was obtained mechanically (bagasse), and the other part was obtained manually (peel). The bagasse and peel were dehydrated at $40^{\circ} \mathrm{C}$ and the extracts were
\end{abstract}


prepared using a 1:10 (w/v) ratio of $70 \%$ ethanol. After homogenization, the extracts were vacum filtered and rotaevaporizedat $40{ }^{\circ} \mathrm{C}$ and $50{ }^{\circ} \mathrm{C}$. It was verified that the extraction of total phenolic compounds, flavonoids and anthocyanins was significantly higher at $50^{\circ} \mathrm{C}$. When comparing the two types of residues, the peel presented higher levels of flavonoids, anthocyanin, antioxidant (DPPH and FRAP) and antimicrobial activity, although for the total phenolic compounds no significant difference was observed between the types of residues. Concerning the antimicrobial capacity, it was observed that the extracts were effective against Listeria monocytogenes, Bacilus cereus and Staphylococcus aureus, being the peel the most efficient. Thus, the peel extracts rotaevaporate at $50^{\circ} \mathrm{C}$ can be considered a potential research source to bring nutritional benefits, as well as for the reduction of residues in the environmental impact, being able to be incorporated in new products by the food, pharmaceutical and related industries.

Keywords: Peel; Bagasse; Antioxidant activity; Antimicrobial activity.

\section{Resumen}

El jamelão es una fruta rica en compuestos bioactivos y poco utilizada por la industria. Dado que no existe estudio sobre el efecto de la temperatura de rotaevaporización y el tipo de residuo en la extracción de bioactivos de esta fruta. El objetivo de este trabajo fue verificar la influencia de estos factores en su extracción en la actividad antioxidante y antimicrobiana. Los residuos se obtuvieron de forma mecánica (bagazo) y la otra parte de forma manual (cáscara). El bagazo y la cáscara se deshidrataron a $40^{\circ} \mathrm{C}$ y los extractos se prepararon usando una proporción $1: 10(\mathrm{p} / \mathrm{v})$ de alcohol etílico al $70 \%$. Después de la homogeneización, los extractos se filtraron al vacío y se rotavaporaron a $40^{\circ} \mathrm{C}$ y $50^{\circ} \mathrm{C}$. Se pudo comprobar que la extracción del contenido de fenólicos, flavonoides y antocianinas fue significativamente mayor a $50^{\circ} \mathrm{C}$. En relación a la comparación entre los tipos de residuos, la cáscara fue la mejor fuente extractora para los niveles de flavonoides, antocianina, actividad antioxidante (DPPH y FRAP) y actividad antimicrobiana, aunque para los fenólicos no se observó diferencia significativa entre tipos de residuos. En cuanto a la capacidad antimicrobiana, se observó que los extractos fueron eficaces frente a Listeria monocytogenes, Bacilus cereus y Staphylococcus aureus, siendo la cáscara, más eficaz. Así, los extractos de cáscara producidos a $50^{\circ} \mathrm{C}$ pueden considerarse una fuente potencial de estudios para aportar beneficios nutricionales, así como para reducir los residuos en el impacto ambiental, y pueden ser incorporados en nuevos productos por la industria alimentaria, farmacéutica y afines.

Palabras clave: Cáscara; Bagazo; Actividad antioxidante; Actividad antimicrobiana.

\section{Introdução}

O consumo de frutas tropicais no Brasil vem aumentando graças aos estudos realizados que reconhecem seus valores nutricionais e terapêuticos, dentre elas destaca-se o jamelão (Santiago, et al., 2016). O jamelão é conhecido em diversas regiões do Brasil, visto que é uma planta adaptada a diferentes condições climáticas, possibilitando o aparecimento em estados da região norte, nordeste e sudeste; apresentando diversos nomes como: jamelão, jambolão, azeitona-roxa e oliva (Corrêa, et al., 2018).

Embora seja um fruto rico em compostos bioativos, a maior parte desses frutos é perdida no seu período de safra, pois a árvore apresenta uma alta produção em um curto período de tempo, agregado ao fato de ser um fruto altamente perecível e pouco utilizado pela indústria (Barcia, 2009).Existem na literatura alguns trabalhos que utilizam resíduos de frutos (cascas e sementes) como matéria-prima para obtenção de compostos bioativos com propriedades antioxidantes agregando assim valor a este material que seria descartado (Almeida, et al., 2020; Aquino, et al.; 2020).

Devido à presença dos compostos bioativos, o jamelão apresenta alta atividade antioxidante, sendo uma fonte rica em antocianinas, delfinidina-3-glicosídeo, petunidina-3-flicosídeo e malvidina-3-glicosídeo (Vizzoto, et al., 2008).

Diferentes partes desse fruto (polpa, casca e semente) também foram estudadas por outros autores, os quais identificaram potencial antioxidante, atividade antidiabética, antifúngicas e antibacterianas. Além de outros compostos fenólicos, flavonóides, carotenóides e ácido elágico, fornecendo outras propriedades funcionais (Ayyanar \& Subash-Babu, 2012; Sari, et al., 2012; Neves, 2017).

Além disso, o jamelão tem sido muito estudado quanto à atividade antibacteriana uma vez que alguns autores demonstraram esses efeitos na folha (Kaneria, et al., 2009), no óleo essencial da folha (Shafi, et al., 2002), nas sementes (Acharyya, et al.,2009), na farinha do fruto (Migliato, et al., 2011) e farinha da casca (Neves, 2017).

A extração de compostos bioativos é influenciada pelo tipo e concentração do solvente, temperatura e tempo de secagem e de extração, tamanho das partículas e metodologia aplicada (Escriche, et al., 2018). Dentre as diversas técnicas utilizadas, os métodos ultrassônicos vêm sendo aplicados para a extração de compostos bioativos de vários materiais, essa técnica é 
considerada um processo verde e sua eficácia vem da comprovação na extração de diferentes antioxidantes, tendo um maior rendimento, com economia de tempo e energia (Oroian, et al., 2020). A irradiação causada pelo ultrassom, ajuda na aceleração das etapas de homogeneização e transferência de massa entre fases diferentes, além de que, quando comparado com outros métodos, o aparelho possui um custo mais baixo e de fácil operação (Asfaram, et al., 2017; Wang, et al., 2006).

Não existe um solvente ideal para a extração de compostos bioativos, pois devido à grande diversidade, nenhum solvente tem a capacidade de isolar todos, principalmente nos vegetais que apresentam uma grande quantidade e variedade dos compostos (Savi, 2015). O principal solvente utilizado para extrações de compostos bioativos de plantas é o etanol em diferentes concentrações, devido sua atoxidade e alta eficiência de extração de polifenóis (Oroian, et al., 2020).

A temperatura também é um fator importante no processo de extração, caso esteja elevada aumenta-se as taxas de difusão e solubilidade dos analitos, por outro lado ocorre uma diminuição na viscosidade e na tensão superficial dos solventes. Essas condições aceleram o processo de extração por facilitar a interação dos analitos com os solventes, entretanto pode-se observar uma diminuição no rendimento do processo, visto que alguns compostos podem ser degradados, caso a exposição a essas temperaturas seja prolongada (Oliveira, et al., 2014).

Visto que não existe literatura sobre o efeito da temperatura de rotaevaporação e do tipo de resíduo na extração de compostos bioativos, o objetivo deste trabalho foi verificar a influência desses fatores na extração destes compostos, e consequentemente nas atividades antioxidante e antimicrobiana.

\section{Metodologia}

Nesse estudo foi utilizada metodologia quantitativa realizada de acordo os métodos propostos por Thaipong et al. (2006) e Boroski et al. (2015).

\section{Matéria-prima}

Os frutos foram obtidos no mercado central e foram inicialmente sanitizados com hipoclorito de sódio a 200 ppm por 30 minutos com subsequente enxágue por 10 minutos. Em seguida, uma parte da amostra foi obtida mecanicamente em despolpadora industrial (Itametal, modelo compacto) originando o bagaço; a outra parte foi descascada manualmente com o auxílio de uma faca resultando no resíduo denominado casca. O bagaço e a casca foram desidratados a $40{ }^{\circ} \mathrm{C}$ em estufa com circulação de ar tipo pardal (Pardal, modelo PE 100) por aproximadamente 20 horas, até umidade final de 7-10 \%. Por fim após a secagem, o bagaço e a casca foram processados separadamente em moinho de pás (Tecnal, modelo TE 650) a fim da obtenção das farinhas, que foram armazenadas em embalagens laminadas, protegidas da luz, sob vácuo e congeladas a $-18{ }^{\circ} \mathrm{C}$ até o momento do uso.

\section{Reagentes químicos}

Todos os produtos químicos e reagentes utilizados para determinação dos compostos bioativos e da atividade antioxidante total das amostras foram de qualidade analítica. Os reagentes: Folin-Ciocalteu fenol, ácido gálico (GA); radical 2,20-azino-bis (ácido 3-etilbenzenotiazolina-6-sulfônico) (ABTS); 2,4,6 tripiridil-s-triazina (TPTZ), persulfatode potássio, sulfato ferroso, ácido 6-hidroxi-2,5,7,8-tetrametilcroman-2-carboxílico (Trolox), quercetina e cloreto férrico foram da SigmaAldrich (EUA), enquanto a acetona, ácido clorídrico $(\mathrm{HCl})$, carbonato de sódio e cloreto de alumínio foram da Dynamic ou Neon (Brasil).

\section{Obtenção dos Extratos}

Para a preparação dos extratos foi utilizado uma proporção de 1:10 (m:v) de álcool etílico $70 \%$ e a solução foi 
homogeneizada por 1 hora em banho de ultrassom (Excel, modelo Alpha 3L plus) sem interferência da luz. Após a homogeneização, os extratos foram filtrados a vácuo e submetidos a concentração por rotaevaporação (Buchi, modelo R-3) em pressão reduzida em duas temperaturas $\left(40{ }^{\circ} \mathrm{C}\right.$ e $\left.50{ }^{\circ} \mathrm{C}\right)$ até completa evaporação do solvente. Os extratos prontos foram armazenados em frasco âmbar e congelados até o uso.

\section{Quantificação dos Compostos Bioativos}

Os compostos fenólicos totais foram quantificados de acordo com o procedimento convencional espectrofotométrico de Folin-Ciocalteau modificado por Thaipong et al. (2006), utilizando ácido gálico como padrão. Os flavonoides foram quantificados conforme o método descrito por Boroski et al. (2015) com algumas modificações, utilizando quercetina como padrão. O teor de antocianina monomérica total (TMA) foi quantificado utilizando o método de $\mathrm{pH}$ diferencial que se baseia na transformação estrutural do cromóforo de antocianina em função do pH. Esse método foi citado por Santos et al. (2010) e descrito por Giusti e Wrolstad (2001), com algumas modificações.

\section{Quantificação da Atividade Antioxidante}

A avaliação da atividade antioxidante total foi realizada pelos métodos de captura dos radicais ABTS e DPPH e também foi avaliada pelo potencial redutor do ferro nos extratos, pelo teste FRAP. Foram utilizados como padrões de referência antioxidante, o trolox e a solução de sulfato ferroso. As três metodologias seguiram os procedimentos descrito por Boroski et al. (2015).

\section{Determinação da Atividade Antimicrobiana}

A avaliação da atividade antimicrobiana dos extratos foi realizada pelo método de disco-difusão em ágar proposto por Jing-Em et al. (2015), no qual foram utilizadas sete cepas de microrganismos variando entre bactérias gram-positivas (Staphylococcus aureus ATCC 6538, Listeria monocytogenes ATCC 7644, Bacillus cereus ATCC 11778) e gram-negativas (Salmonella Enteritidis ATCC 13076, Salmonella Typhimurium ATCC 14028, Escherichia coli ATCC 8739, Escherichia coli O157:H7 ATCC 35150), um controle positivo (clorexidina 0,12\%) e outro negativo (tampão pH 7). A zona de inibição (ZI) do crescimento microbiano foi avaliada pelo diâmetro do halo de inibição em $\mathrm{mm}$ ao redor dos discos após 24 horas de incubação a $37 \pm 1{ }^{\circ} \mathrm{C}$. Os ensaios foram realizados em triplicatas, e os resultados expressos pela média aritmética dos valores dos halos obtidos nas repetições.

\section{Análise estatística}

A comparação de médias das amostras para cada análise e para cada tipo de resíduo foi realizada através da análise de variância e teste Tukey a 5\% de significância, por meio do software Sisvar.

\section{Resultados e Discussão}

Após a desidratação dos resíduos de jamelão, o rendimento foi de $20 \%$ tanto para as cascas quanto para o bagaço. O pó apresentou coloração roxa intensa atraente, e embora a taxa de rendimento não tenha sido alta, qualquer aproveitamento dos resíduos dessa fruta é vantajoso, pois além de serem ricos em compostos bioativos, geralmente são desperdiçados durante a safra, visto que são pouco utilizados pela indústria de alimentos.

\section{Compostos bioativos}

Nas Tabelas 1 e 2 é possível verificar os resultados obtidos na quantificação de compostos bioativos presentes nos 
resíduos de jamelão.

Tabela 1. Quantificação de compostos fenólicos totais dos extratos da casca e do bagaço de jamelão (Syzygium cumini) rotaevaporados em temperaturas de $40{ }^{\circ} \mathrm{C}(\mathrm{T} 40)$ e $50^{\circ} \mathrm{C}(\mathrm{T} 50)$.

\begin{tabular}{lcc}
\hline \multirow{2}{*}{ Amostra } & \multicolumn{2}{c}{ Compostos Fenólicos Totais (mg GAE.g $\left.{ }^{-1}\right)$} \\
\cline { 2 - 3 } & T40 & T50 \\
\hline Casca & $67,66 \pm 3,48 \mathrm{bA}$ & $90,98 \pm 4,24 \mathrm{aA}$ \\
Bagaço & $47,29 \pm 3,08 \mathrm{bB}$ & $81,35 \pm 3,75 \mathrm{aA}$ \\
\hline
\end{tabular}

As análises foram realizadas em triplicata e os resultados estão expressos pela média aritmética entre 3 a 5 replicatas. Letras maiúsculas iguais não diferem significativamente na mesma coluna e letras minúsculas iguais não diferem significativamente na mesma linha, segundo teste Tukey $(\mathrm{p}<0,05)$. Fonte: Autores.

Verifica-se na Tabela 1 que enquanto a rotaevaporação a $40{ }^{\circ} \mathrm{C}$ na casca extraiu $67,66 \mathrm{mg} \mathrm{GAE} \cdot \mathrm{g}^{-1}$ de compostos fenólicos, a rotaevaporação a $50{ }^{\circ} \mathrm{C}$ conseguiu extrair uma quantidade ainda maior desses compostos $\left(90,98 \mathrm{mg}\right.$ GAE.g $\left.{ }^{-1}\right)$. No bagaço também foi observado um aumento na extração com o aumento da temperatura de rotaevaporação, verificando uma extração 1,7 vezes maior para o bagaço rotaevaporado a $50^{\circ} \mathrm{C}$ quando comparado ao rotaevaporado a $40{ }^{\circ} \mathrm{C}\left(47,29 \mathrm{mg}\right.$ GAE.g ${ }^{-1}$ e $81,35 \mathrm{mg} \mathrm{GAE} \cdot \mathrm{g}^{-1}$ para os extratos rotaevaporados a $40{ }^{\circ} \mathrm{C}$ e $50{ }^{\circ} \mathrm{C}$, respectivamente). Assim percebe-se que o aumento da temperatura de rotaevaporação de $40^{\circ} \mathrm{C}$ para $50^{\circ} \mathrm{C}$ aumentou significativamente $(\mathrm{p}<0,05)$ a quantidade de compostos fenólicos extraídos tanto no bagaço quanto na casca.

Entretanto, ao se comparar a influência dos tipos de resíduos na extração dos compostos fenólicos, observa-se ainda na

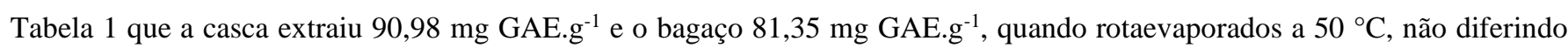
estatisticamente entre si. Por outro lado, a temperatura de $40^{\circ} \mathrm{C}$ influenciou significativamente na extração, pois mais compostos

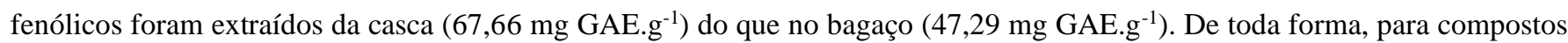
fenólicos, a rotaevaporação a $50{ }^{\circ} \mathrm{C}$ quando comparada a de $40{ }^{\circ} \mathrm{C}$ foi muito mais eficiente, tendo um aumento de $33 \%$ na extração desses compostos presentes na casca e de $70 \%$ para os compostos presentes no bagaço.

Neves (2017), em condições semelhantes ao deste trabalho, para a farinha da casca reportou valores de $36,50 \mathrm{mg} \mathrm{GAE} / \mathrm{g}$ para extrato rotaevaporado a $40^{\circ} \mathrm{C}$, bem como Borges (2011) que relatou teor de 2880,7 mg GAE /100 g para bagaço de jamelão desidratado em leite de jorro, ambos extraídos com etanol $70 \%$, sendo estes valores ainda inferiores aos obtidos no presente trabalho. Estas diferenças podem ser devido a diversos fatores como, a umidade do produto, o tipo de solo, o clima durante o desenvolvimento do fruto (Djeridane, et al., 2013), variação no grau de maturação (Biegelmeyer, et, al., 2011), diferenças genéticas e condições de armazenamento pós-colheita bem como a localização geográfica dos frutos (Cheng, et al., 2012).

Frauches (2017) reportou em seu manuscrito um teor de fenólicos de 852,17 mg GAE/100 g para farinha de casca de jamelão desidratada a $60^{\circ} \mathrm{C}$, valor dez vezes inferior ao da presente pesquisa, provavelmente em função da temperatura de secagem utilizada ser maior do que a deste trabalho $\left(40^{\circ} \mathrm{C}\right)$.

De acordo com os dados apresentados na Tabela 1, é possível constatar que a casca e o bagaço do jamelão são ricos em compostos fenólicos, pois ao comparar com o teor presente em outras frutas normalmente consumidas no Brasil, verifica-se que o jamelão possui um teor de quatro a oito vezes maior $(4270-8700 \mathrm{mg} / 100 \mathrm{~g})$ que a jabuticaba $(1405,79 \mathrm{mg} / 100 \mathrm{~g})$, de oito a 16 vezes maior que a polpa de acerola $(580,1 \mathrm{mg} / 100 \mathrm{~g})$ e de dez a 20 vezes maior em comparação a seriguela(427,12 mg/100 g) e ao cajá $(400,96 \mathrm{mg} / 100 \mathrm{~g})$, respectivamente. Essa diferença pode ser ainda maior quando comparado com o umbu (52,72 mg/100 g), polpa de uva (117,1 mg/100 g) e polpa de cupuaçu (20,5 mg/100 g), aproximadamente 30 vezes maior (Rezende, 2010). 
Pacheco (2015) também reportou que o jamelão foi o fruto com maior teor de compostos fenólicos dentre dez frutos analisados, confirmando a funcionalidade deste fruto.

O alto teor de compostos fenólicos encontrados nesse trabalho pode ser justificado pelo tipo de solvente utilizado, pois a extração do conteúdo fenólico é maior em extratos polares (metanol/etanol) do que em extratos menos polares (Mohamed, et al., 2013; Al-Reza et al., 2009).

Os flavonóides é o maior grupo entre os fenólicos das plantas, que têm sido descritos como tendo muitos benefícios para a saúde devido às suas propriedades antioxidantes, cardio-protetoras e anticancerígenas (Celli, et al., 2011).

Tabela 2. Quantificação flavonoides totais e antocianinas monoméricas totais dos extratos da casca e do bagaço de jamelão (Syzygium cumini) rotaevaporados em temperaturas de $40{ }^{\circ} \mathrm{C}(\mathrm{T} 40)$ e $50^{\circ} \mathrm{C}(\mathrm{T} 50)$.

\begin{tabular}{|c|c|c|c|c|}
\hline \multirow{2}{*}{ Amostra } & \multicolumn{2}{|c|}{ Flavonoides Totais (mg QE. $\mathrm{g}^{-1}$ ) } & \multicolumn{2}{|c|}{$\begin{array}{l}\text { Antocianinas Totais Monoméricas (mg } \\
\text { CE. } \mathrm{L}^{-1} \text { ) }\end{array}$} \\
\hline & $\mathrm{T} 40$ & T50 & $\mathrm{T} 40$ & T50 \\
\hline Casca & $1,77 \pm 0,07 \mathrm{bA}$ & $2,31 \pm 0,09 \mathrm{aA}$ & $886,49 \pm 1,85 \mathrm{bA}$ & $905,91 \pm 7,42 \mathrm{aA}$ \\
\hline Bagaço & $0,90 \pm 0,02 \mathrm{aB}$ & $1,03 \pm 0,12 \mathrm{aB}$ & $347,89 \pm 7,42 \mathrm{aB}$ & $158,63 \pm 9,13 \mathrm{bB}$ \\
\hline
\end{tabular}

As análises foram realizadas em triplicata e os resultados estão expressos pela média de 3 replicatas. Letras maiúsculas iguais não diferem significativamente na mesma coluna e letras minúsculas iguais não diferem significativamente na mesma linha, segundo teste Tukey ( $\mathrm{p}<0,05$ ). Fonte: Autores (2020).

Na Tabela 2 verifica-se que o teor de flavonoides da casca extraído na rotaevaporação a $40{ }^{\circ} \mathrm{C}$ foi de $1,77 \mathrm{mg}$ QE.g-1 e que houve um aumento significativo de 1,3 vezes quando a temperatura foi aumentada para $50^{\circ} \mathrm{C}\left(2,31 \mathrm{mg} \mathrm{QE}^{-1}\right)$. No bagaço, todavia, não houve diferença estatística na extração desses compostos, independentemente da temperatura de rotaevaporação utilizada $\left(0,90 \mathrm{mg} \mathrm{QE.g}{ }^{-1}\right.$ para $40{ }^{\circ} \mathrm{C}$ e $1,03 \mathrm{mg}$ QE.g ${ }^{-1}$ para $\left.50{ }^{\circ} \mathrm{C}\right)$.

Contudo ao se comparar a influência do tipo de resíduo na extração (Tabela 2) verificou-se que a casca extraiu aproximadamente 2 vezes mais flavonoides $\left(1,77 \mathrm{mg}\right.$ QE.g $\left.{ }^{-1}\right)$ que o bagaço $\left(0,90 \mathrm{mg} \mathrm{QE.g}{ }^{-1}\right)$ na rotaevaporação a $40{ }^{\circ} \mathrm{C}$ e o mesmo ocorreu também na rotaevaporação a $50{ }^{\circ} \mathrm{C}$ em que a casca extraiu $2,31 \mathrm{mg}$ QE.g ${ }^{-1}$ e o bagaço $1,03 \mathrm{mg}$ QE.g ${ }^{-1}$, respectivamente. Logo, observa-se que a extração de flavonoides foi significativamente maior (p<0,05) na casca que no bagaço nas duas temperaturas avaliadas e que quando usada a temperatura de $50{ }^{\circ} \mathrm{C}$ sua extração foi ainda maior $\left(2,31 \mathrm{mg}\right.$ QE.g $\left.{ }^{-1}\right)$.

Bezerra (2015) e Neves (2017) reportaram em seus trabalhos teores de flavonóides totais próximos aos obtidos nessa pesquisa para a casca (1,77-2,35 mg QE/g), os quais foram de 165,4 mg /100 g para a polpa, e de 1,94 mg QE/g para a farinha da casca, respectivamente. Por outro lado, Rai et al. (2011) e Maran et al. (2014) analisando o fruto e a polpa liofilizada, reportaram teor de115 mg /100 g e 109,08 mg QE/100 g, respectivamente, valores mais próximos aos observados para o bagaço no presente estudo.

Ainda para o teor de flavonóides, resultados muito inferiores aos reportados nesse estudo foram relatados para outros frutos, como por Belwal et al. (2016) para polpa de berries desidratada (0,82 mg/100 g), por Costa et al. (2012) para a farinha de araticum desidratada a $40{ }^{\circ} \mathrm{C}(13 \mathrm{mg} / 100 \mathrm{~g})$, por Silva (2014) em sementes de acerola desidratada em secador roto-aerado (3,42 mg rutina/100 g) e por Gardusi et al. (2014) para a casca desidratada e in natura de mexerica (4,60 e 3,07 mg de rutina/100 $\mathrm{g}$, respectivamente).

Em relação à quantificação de antocianinas na casca (Tabela 2), constatou-se que a rotaevaporação a $40{ }^{\circ} \mathrm{C}$ extraiu $886,49 \mathrm{mg} \mathrm{CE}$. $\mathrm{L}^{-1}$ e que quando a temperatura foi aumentada para $50{ }^{\circ} \mathrm{C}$ extraiu-se um teor ainda maior de $905,91 \mathrm{mg} \mathrm{CE}$. $\mathrm{L}^{-1}$. 
Assim percebe-se que o aumento da temperatura de rotaevaporação de $40^{\circ} \mathrm{C}$ para $50{ }^{\circ} \mathrm{C}$ na casca aumentou significativamente $(p<0,05)$ a quantidade de compostos antociânicos extraídos. Por outro lado, no bagaço, observou-se uma redução significativa desses compostos com o aumento da temperatura de rotaevaporação de $40{ }^{\circ} \mathrm{C}$ para $50{ }^{\circ} \mathrm{C}$, passando de $347,89 \mathrm{mg} \mathrm{CE}$. $\mathrm{L}^{-1}$ para 158,63 mg CE. $\mathrm{L}^{-1}$, respectivamente.

$\mathrm{Na}$ avaliação da influência do tipo de resíduo pode-se notar que o extrato da casca apresentou valor aproximadamente três vezes maior $\left(886,49 \mathrm{mg} \mathrm{CE}\right.$. $\left.\mathrm{L}^{-1}\right)$ que o do bagaço $\left(347,89 \mathrm{mg} \mathrm{CE}\right.$. $\left.\mathrm{L}^{-1}\right)$ quando rotevaporados a $40{ }^{\circ} \mathrm{C}$ e oito vezes maior (905,91 mg CE. $\left.\mathrm{L}^{-1}\right)$ que o do bagaço $\left(158,63 \mathrm{mg} \mathrm{CE}\right.$. $\left.\mathrm{L}^{-1}\right)$ quando rotaevaporados a $50{ }^{\circ} \mathrm{C}$. A maior extração dos compostos antociânicos na casca ocorre provavelmente em função da menor quebra das moléculas nesse tipo de resíduo se comparado ao bagaço que se encontrava triturado, já que foi obtido mecanicamente.

Neves (2017), em condições semelhantes ao do presente trabalho, observou valor superior (1269,342 mg/100 g) para o pó da casca, enquanto Frauches (2017) encontrou teor de 575,2 mg/100 g de antocianinas em pó da casca desidratada a $60^{\circ} \mathrm{C}$, comparativamente inferior ao da casca e superior ao bagaço obtido no presente estudo.

Santiago et al (2016), por sua vez, encontrou no pó da casca do jamelão teor de antocianina de 878,56 mg/100 g em amostras desidratadas a $60{ }^{\circ} \mathrm{C}$, resultados próximos aos obtidos neste trabalho para o extrato T50.

A comparação dos valores observados nessa pesquisa com os relatados na literatura foi baseada, em sua maioria, em relação à temperatura de secagem dos resíduos, tendo em vista que até o momento não existem trabalhos relatando o efeito da temperatura de rotaevaporação dos extratos sobre o teor dos compostos bioativos.

No geral, pode-se constatar nas Tabelas 1 e 2 que os fatores que mais influenciaram significativamente na extração foi o tipo de resíduo (casca) e a temperatura de rotaevaporação de $50^{\circ} \mathrm{C}$, sendo esta combinação a que possibilitou maior extração dos teores de flavonoides totais e antocianinas monoméricas, sendo que para os compostos fenólicos não teve diferença significativa entre as amostras, o que leva a crer que a classe de flavonoides sofre mais a influência de danos mecânicos, sendo menos extraída no bagaço.

\section{Atividade Antioxidante}

Os valores das atividades antioxidantes totais pelos métodos de $\mathrm{ABTS}^{+}$, DPPH e FRAP podem ser vistos nas Tabelas 3 e 4.

Tabela 3. Quantificação da atividade antioxidante pelos métodos de captura dos radicais $\mathrm{ABTS}^{+}$e DPPH dos extratos da casca e bagaço de jamelão (Syzygium cumini) rotaevaporados em temperaturas de $40{ }^{\circ} \mathrm{C}(\mathrm{T} 40)$ e $50{ }^{\circ} \mathrm{C}(\mathrm{T} 50)$.

\begin{tabular}{ccccc}
\hline \multirow{2}{*}{ Amostra } & \multicolumn{2}{c}{ ABTS $\left(\mu\right.$ molTE. $\left.{ }^{-1}\right)$} & \multicolumn{2}{c}{ DPPH (EC50\%) } \\
\cline { 2 - 5 } & T40 & T50 & T40 & T50 \\
\hline Casca & $1083,56 \pm 0,015 \mathrm{bB}$ & $1332,19 \pm 0,010 \mathrm{aB}$ & $305,95 \pm 5,34 \mathrm{aA}$ & $140,14 \pm 10,06 \mathrm{aA}$ \\
Bagaço & $1418,06 \pm 0,004 \mathrm{bA}$ & $1864,41 \pm 0,010 \mathrm{aA}$ & $238,60 \pm 56,37 \mathrm{aA}$ & $153,25 \pm 22,73 \mathrm{aA}$
\end{tabular}

As análises foram realizadas em triplicata e os resultados estão expressos pela média aritmética \pm desvio padrão. Letras maiúsculas iguais na mesma coluna não diferem significativamente e letras minúsculas iguais na mesma linha não diferem significativamente, segundo teste Tukey $(\mathrm{p}<0,05)$. Fonte: Autores.

Para a análise de atividade antioxidante pelo método ABTS as amostras diferenciaram-se entre si tanto para o fator temperatura de rotaevaporação como para o fator tipo de resíduo $(\mathrm{p}<0,05)$. Verifica-se na Tabela 3, para esse método, que o aumento da temperatura da rotaevaporação promoveu o aumento da atividade antioxidante tanto para o extrato da casca, quanto para o extrato do bagaço. Pois enquanto a rotaevaporação a $40{ }^{\circ} \mathrm{C}$ na casca apresentou 1083,56 $\mu$ molTE. $\mathrm{g}^{-1}$ de atividade 
antioxidante total, a rotaevaporação a $50^{\circ} \mathrm{C}$ apresentou uma atividade ainda mais alta de $1332,19 \mu$ molTE. ${ }^{-1}$. No bagaço também foi observado um aumento na atividade com o aumento da temperatura de rotaevaporação, verificando uma extração de 1,3 vezes maior para o bagaço rotaevaporado a $50{ }^{\circ} \mathrm{C}$ quando comparado ao rotaevaporado a $40{ }^{\circ} \mathrm{C}\left(1418,06 \mu\right.$ molTE. $\mathrm{g}^{-1} \mathrm{e} 1864,41$ $\mu$ molTE. $\mathrm{g}^{-1}$, respectivamente).

Da mesma forma ao se comparar a influência dos tipos de resíduos na captura dos radicais $\mathrm{ABTS}^{+}$foi visto que na temperatura de rotaevaporação a $40{ }^{\circ} \mathrm{C}$ a casca apresentou uma atividade 1083,56 $\mu$ molTE. ${ }^{-1}$ enquanto que no bagaço foi verificado um aumento de 1,3 vezes $\left(1418,06 \mu\right.$ molTE. $\left.\mathrm{g}^{-1}\right)$, o que também ocorreu na temperatura de $50{ }^{\circ} \mathrm{C}$ em que a casca apresentou atividade de 1332,19 $\mu$ molTE. $\mathrm{g}^{-1}$ e o bagaço de 1,4 vezes maior $\left(1864,41 \mu\right.$ molTE. $\left.\mathrm{g}^{-1}\right)$. Assim, pode-se dizer que o extrato do bagaço T50 apresentou atividade antioxidante pelo método ABTS significativamente melhor que as demais amostras.

Santiago et al (2016) quantificaram a capacidade antioxidante por ABTS em farinha de casca de jamelão desidratada a $60{ }^{\circ} \mathrm{C}$ em secador de bandejas e reportaram $150 \mu \mathrm{MTE} . \mathrm{g}^{-1}$, assim como Rufino et al. (2010) que também avaliaram farinha da casca+polpa de jamelão, porém desidratadas por liofilização e obtiveram $125 \mu \mathrm{MTE} . \mathrm{g}^{-1}$, por outro lado, Constancio (2015) reportou 71,58 $\mu \mathrm{MTE} . \mathrm{g}^{-1}$ também para polpa de jamelão liofilizada. Verifica-se que nestas pesquisas os valores foram inferiores aos reportados no presente trabalho, provavelmente em virtude do método, temperatura de secagem utilizada ser maior do que a do presente trabalho $\left(40{ }^{\circ} \mathrm{C}\right)$ ou até mesmo em função do tipo de solvente utilizado para a obtenção dos extratos, pois nos trabalhos acima os extratos foram elaborados com metanol: água e acetona: água e neste trabalho foi usado etanol: água.

Por outro lado, Frauches (2017) observou valores semelhantes ao relatado na presente pesquisa $(1083,56$ - 1332,19 $\mu \mathrm{MTE} . \mathrm{g}^{-1}$ ) para o pó desidratado da casca de jamelão onde reportou um valor de 1038,50 \pm 7,22 $\mu$ MTE.g ${ }^{-1}$. Todavia, Neves (2017) determinou em um extrato da farinha da casca obtido em condições semelhantes ao presente estudo e rotaevaporado a $40{ }^{\circ} \mathrm{C}$ o teor de $921,03 \mu \mathrm{MTE} . \mathrm{g}^{-1}$.

Para a atividade quantificada pelo método de captura do radical DPPH não foi observada diferença significativa para nenhum dos fatores analisados (temperatura de rotaevaporação ou tipo de resíduo), todavia foi observado menores valores de EC $50 \%$ para a temperatura de rotaevaporação de $50^{\circ} \mathrm{C}$, tanto para a casca $(140,14)$ como para o bagaço $(153,25)$ o que significa dizer que os extratos T50 utilizaram uma menor concentração de compostos antioxidantes para reduzir o radical a 50\% de sua atividade, logo eles apresentaram melhores resultados, especialmente o da casca, mesmo que não significativo.

Por outro lado, Neves (2017) encontrou valores muito inferiores de EC $50 \%$ para o pó da casca desidratado a $40{ }^{\circ} \mathrm{C}$ $(9,96)$, enquanto Rufino et al. (2010) para jamelão (casca+polpa) desidratado por liofilização reportaram valores muito superiores (938), como já mencionado, essa diferença na atividade antioxidante pode ser explicada provavelmente em virtude da diferença dos solventes utilizados.

Esses resultados demonstram que a maior quantidade dos compostos antioxidantes reage melhor aos radicais ABTS, pois envolvem um processo de transferência de elétrons, sendo portanto mais reativos do que os radicais DPPH que podem envolver tanto a transferência de elétrons como a transferência de átomos de hidrogênio (Gulcin, 2012; Huang \& Prior, 2005). 
Tabela 4. Quantificação da atividade antioxidante pelo método FRAP dos extratos da casca e bagaço de jamelão (Syzygium cumini) rotaevaporados em temperaturas de $40{ }^{\circ} \mathrm{C}(\mathrm{T} 40)$ e $50{ }^{\circ} \mathrm{C}(\mathrm{T} 50)$.

\begin{tabular}{lcc}
\hline Amostra & \multicolumn{2}{c}{$\mathrm{FRAP}\left(\mu \mathrm{molFe} \mathrm{F}^{2+} \cdot \mathrm{g}^{-1}\right)$} \\
\cline { 2 - 3 } & $\mathrm{T} 40$ & $\mathrm{~T} 50$ \\
\hline Casca & $2220,80 \pm 3,64 \mathrm{aA}$ & $2623,66 \pm 81,88 \mathrm{aA}$ \\
Bagaço & $1320,89 \pm 25,37 \mathrm{bB}$ & $1453,69 \pm 9,42 \mathrm{aB}$
\end{tabular}

As análises foram realizadas em triplicata e os resultados estão expressos pela média aritmética. Letras maiúsculas iguais na mesma coluna não diferem significativamente e letras minúsculas iguais na mesma linha não diferem significativamente, segundo teste Tukey (p<0,05). Fonte: Autores.

Analisando a temperatura de rotaevaporação na atividade antioxidante total pelo método FRAP (Tabela 4) verificou-se para o extrato da casca um aumento não significativo com o aumento da temperatura de rotaevaporação de $2220,80 \mu$ molFe ${ }^{2+}$.g ${ }^{1}$ a $40{ }^{\circ} \mathrm{C}$ para $2623,66 \mu \mathrm{molFe}^{2+} \cdot \mathrm{g}^{-1}$ a $50{ }^{\circ} \mathrm{C}$. Todavia para o bagaço, o aumento da temperatura resultou em um extrato com atividade antioxidante significativamente maior $\left(1320,89 \mu \mathrm{molFe} e^{2+} \cdot \mathrm{g}^{-1} \mathrm{e} 1453,69 \mu \mathrm{molFe} \mathrm{F}^{2+} \cdot \mathrm{g}^{-1}\right.$ para os extratos rotevaporados a 40 e $50^{\circ} \mathrm{C}$, respectivamente).

Ao se analisar a influência do tipo de resíduo na atividade antioxidante ainda na Tabela 4, percebeu-se que a casca apresentou resultado aproximadamente duas vezes superior ao do bagaço tanto para temperatura de rotaevaporação a $40{ }^{\circ} \mathrm{C}$ $\left(2220,80 \mu \mathrm{molFe} e^{2+} \cdot \mathrm{g}^{-1}\right.$ para a casca e $1320,80 \mu \mathrm{molFe}{ }^{2+}$ para o bagaço $)$ como para a temperatura a $50^{\circ} \mathrm{C}\left(2623,81 \mu \mathrm{molFe}{ }^{2+} . \mathrm{g}^{-1}\right.$ para casca e 1453,69 $\mu \mathrm{molFe}^{2+} . \mathrm{g}^{-1}$ para o bagaço), mostrando que o tipo de resíduo influenciou significativamente na atividade antioxidante dos extratos. Logo, dentre todas as amostras, a que apresentou melhor resultado foi o extrato elaborado com a casca rotaevaporado a $50{ }^{\circ} \mathrm{C}$.

Os resultados obtidos pela metodologia FRAP na presente pesquisa comprovam a alegação de Mohamed et al (2013), que afirmam que a capacidade de reduzir o Fe (III)é atribuída à doação de hidrogênio pelo composto fenólico que está relacionado à presença de um agente redutor, já que verificou-se que as maiores atividades antioxidantes estavam presentes nos extratos elaborados com a casca, que foi o resíduo com maior quantidade de compostos bioativos (Tabela 1).

Fraches (2017) ao avaliar a atividade antioxidante de casca de jamelão desidratado a $60{ }^{\circ} \mathrm{C}$, pelo método de FRAP, constatou valor médio de 702,42 $\mu \mathrm{mol}$ de sulfato ferroso/g, enquanto Rufino et al (2010) reportaram valor de $173 \mu \mathrm{mol}$ de sulfato ferroso/g em jamelão (casca+polpa) liofilizado. Verifica-se que ambos os valores foram inferiores ao do presente trabalho, provavelmente em função da diferença na temperatura de secagem e do tipo de solvente de extração. Todavia Neves (2017) relatou um valor muito superior ( $7042 \mu \mathrm{mol}$ de sulfato ferroso/g) para o extrato da casca, mesmo em condições semelhantes ao da presente pesquisa, o que pode ser justificado pelas condições climáticas em que os frutos foram produzidos.

No geral percebe-se que os extratos elaborados com a casca e rotaevaporados a $50{ }^{\circ} \mathrm{C}$ foram os que apresentaram maior atividade antioxidante, exceto para aqueles obtidos pela metodologia de $\mathrm{ABTS}^{+}$.

\section{Atividade Antimicrobiana}

Os resultados da atividade antimicrobiana mostraram que independentemente do tipo de resíduo e da temperatura de rotaevaporação, os extratos só foram eficientes contra Listeria monocytogenes (LM), Staphylococcus aureus (SA) e Bacillus cereus (BC), cujos diâmetros (mm) da zona de inibição encontram-se na Tabela 5. 
Tabela 5. Análise de atividade antimicrobiana pelo método disco-difusão $(\mathrm{mm})$ dos extratos da casca e bagaço de jamelão (Syzygium cumini) rotaevaporados em temperaturas de $40^{\circ} \mathrm{C}$ e $50^{\circ} \mathrm{C}$.

\begin{tabular}{cccc}
\hline AMOSTRA & LM & SA & BC \\
\hline cascaT40 & $9,67 \pm 0,57 \mathrm{a}$ & $13,66 \pm 1,52 \mathrm{ab}$ & $8,0 \pm 0,00 \mathrm{~b}$ \\
casca T50 & $12,00 \pm 1,00 \mathrm{a}$ & $15,33 \pm 2,12 \mathrm{ab}$ & $9,5 \pm 0,70 \mathrm{ab}$ \\
bagaço T40 & $9,33 \pm 1,52 \mathrm{a}$ & $11,66 \pm 2,08 \mathrm{~b}$ & $8,5 \pm 0,70 \mathrm{~b}$ \\
bagaço T50 & $10,33 \pm 0,57 \mathrm{a}$ & $11,00 \pm 0,00 \mathrm{~b}$ & - \\
controle positivo & $11,50 \pm 0,71 \mathrm{a}$ & $19,5 \pm 4,50 \mathrm{a}$ & $10,5 \pm 0,70^{\mathrm{a}}$
\end{tabular}

LM: Listeria monocytogenes, SA: Staphylococcus aureus, BC: Bacillus cereus, -: Não houve formação de halo. As análises foram realizadas em triplicata e os resultados estão expressos pela média \pm desvio padrão. Letras iguais não diferem significativamente na mesma coluna $(\mathrm{p}<0,05)$, segundo teste Tukey. Fonte: Autores.

$\mathrm{Na}$ Tabela 5 pode-se verificar que o tipo de resíduo ou a temperatura de rotaevaporação não influenciaram significativamente na inibição de L. monocytogenes, e embora não significativo, o extrato que apresentou melhor resultado foi o da casca rotaevaporado a $50^{\circ} \mathrm{C}(12 \mathrm{~mm})$, resultado semelhante ao do controle positivo $(11,50 \mathrm{~mm})$.

Foi possível constatar que o S. aureus foi o microrganismo mais sensível aos extratos testados quando comparado à sensibilidade das demais bactérias. O extrato elaborado com o bagaço, independente da temperatura de rotaevaporação (11 mm para T40 e 11,66 mm para T50), foi significativamente menos eficiente para o controle desse microrganismo quando comparado com controle positivo clorexidina $0,12 \%(19,5 \mathrm{~mm})$. E embora o tipo de resíduo e a temperatura de rotaevaporação não tenham influenciado significativamente na inibição desse microrganismo, os extratos da casca foram os que formaram os maiores halos de inibição (13,66 mm e 15,33 mm para T40 e T50, respectivamente).

Para B. cereus, no entanto, apenas o extrato da casca a $50{ }^{\circ} \mathrm{C}(9,5 \mathrm{~mm})$ não diferiu significativamente do controle positivo (10,5 mm), os demais resíduos (casca T40, bagaço T40) foram significativamente menos eficientes, ou seja, formaram zonas de inibição significativamente menores. Todavia, ainda assim, a casca a $50{ }^{\circ} \mathrm{C}$ foi a que apresentou melhor atividade antimicrobiana $(9,5 \mathrm{~mm})$. Nota-se ainda na Tabela 5 que não foi observada atividade para o extrato T50 elaborado com o bagaço.

No geral percebe-se que para atividade antimicrobiana, os extratos que apresentaram melhor atividade frente aos microrganismos avaliados foram os elaborados com a casca e rotaevaporados a $50{ }^{\circ} \mathrm{C}$, embora nem sempre significativamente melhor.

Neves (2017) verificou que o extrato da farinha da casca do jamelão testado frente a sete micro-organismos foi eficiente apenas para Staphylococcus aureus e Listeria monocytogenes. De acordo com a literatura em geral, as bactérias Gram-positivas são mais sensíveis do que as Gram-negativas devido a presença de uma membrana externa envolvendo a parede celular das gram-negativas, o que restringe a difusão de compostos hidrofóbicos através de sua cobertura lipopolissacarídica (Alboofetileh, et, al., 2014; Sanchez-Gonzalez, et al., 2011).

Migliato et al. (2010) avaliaram a extração de compostos com atividade antimicrobiana da farinha do fruto jamelão desidratados a $40^{\circ} \mathrm{C}$ em estufa com circulação de ar, frente a Staphylococcus aureus, Staphylococcus epidermidis, Pseudomonas aeruginosa, Enterococcus faecalis, Escherichia coli, Candida albicans, Candida parapsilosis e Candida krusei, e também verificaram maiores zonas de inibição para os microrganismos gram-positivos, com diâmetros dos halos de inibição frente a $S$. aureus variando de 0 a 4,8 mm, sendo estes inferiores aos constatados nesse estudo. Além disso, verificaram também que não foi encontrada atividade antimicrobiana frente aos micro-organismos gram-negativos, assim como no presente trabalho.

Correa et al. (2018) verificaram que extratos etanoicos a $70 \%$ da casca da árvore, das folhas e das sementes de jamelão 
inibiram o crescimento de Escherichia coli $(7$ a $10 \mathrm{~mm})$, diferente deste trabalho que não observou inibição para este microrganismo, independente do extrato utilizado, provavelmente em função do tipo de matéria-prima utilizada para a elaboração dos extratos, mesmo pertencendo à espécie Syzygium cumini.

Mothana e Lindequist (2005) classificaram os extratos em função dos tamanhos dos halos de inibição e afirmaram que extratos que formam halos de inibição entre 8 a $13 \mathrm{~mm}$ devem ser considerados com poder de ação moderadamente ativos, já halos de inibição > $14 \mathrm{~mm}$ são considerados extratos muito ativos. Diante dessa classificação os extratos T40 e T50 tanto da casca quanto do bagaço do jamelão podem ser classificados com atividade antimicrobiana moderada frente às bactérias Listeria monocytogenes e Bacillus cereus, por outro lado os extratos T50 produzido com a casca pode ser classificado com atividade antimicrobiana muito ativa frente a $S$. aureus.

Esses resultados corroboram com os estudos de Martins (2011) que afirmou que a ação antimicrobiana dos extratos se deve à presença de compostos fenólicos, e isso pode ser uma explicação para a maior atividade do extrato T50 da casca, afinal ele obteve os maiores teores dos compostos bioativos analisados.

\section{Conclusão}

Pode-se concluir que a temperatura de rotaevaporação influenciou significativamente a extração dos compostos bioativos, pois os extratos dos diferentes resíduos de jamelão rotaevaporados a $50{ }^{\circ} \mathrm{C}$ apresentaram-se com maiores atividades antioxidantes e antimicrobianas, especialmente o que foi elaborado com a casca. Dessa forma este extrato pode ser considerado uma fonte potencial de estudos para trazer benefícios nutricionais, como também para a redução do impacto ambiental pela redução de resíduos gerados, podendo ser acrescentado em novos produtos pela indústria alimentícia, farmacêutica e afins.

Sugere-se então, em trabalhos futuros, verificar a influência da temperatura de secagem dos resíduos nos teores de compostos bioativos, bem como a incorporação desses extratos em diferentes produtos.

\section{Agradecimentos}

Os autores agradecem à CAPES pelo financiamento da pesquisa e aos coordenadores dos laboratórios de Análises de Alimentos, Bioquímica de Alimentos e de Biotecnologia do Departamento de Tecnologia de Alimentos da Universidade Federal de Sergipe pelos auxílios durante a execução do projeto.

\section{Referências}

Acharyya, S., Patra, A., \& Bag, P. K. (2009). Evaluation of antimicrobial activity of some medicinal plants against enteric bacteria with particular reference to multi-drug resistant vibrio cholera. Tropical Journal of Pharmaceutical Research, 8 (3), 231-237. https://doi.org/0.4314/tjpr.v8i3.44538.

Alboofetileh, M., Rezaei, M., Hosseini, H., \& Abdollahi, M. (2014). Antimicrobial activity of alginate/clay nanocomposite films enriched with essential oils against three common foodborne pathogens. Food Control, 36 (1), 1-7. https://doi.org/10.1016/j.foodcont.2013.07.037.

Almeida, R. L., Santos, N. C., Pereira, T. dos S., Silva, V. M. de Alcântara, Cabral, M. B., Barros, E. R., Souza, N. C. de, Luiz, M. R., Amorim, F. V., \& Silva, L. R. I. da. (2020). Determination of bioactive compounds and physicochemical composition of jabuticaba bark flour obtained by convective drying and lyophilization. Research, Society and Development, 9 (1), e157911876. https://doi.org/10.33448/rsd-v9i1.1876.

Al-Reza, S. M., Rahman, A., \& Kang, S. C. (2009). Chemical composition and inhibitory effect of essential oil and organic extracts of Cestrum nocturnum L. on food-borne pathogens. International Journal of Food Science \& Technology, 44 (6), 1176-1182. https://doi.org/10.1111/j.1365-2621.2009.01939.x. Aquino, T. A. C., Gualberto, N. C., Narain, N., \& Santana, L. C. L. de A. (2020). Evaluation of bioactive compounds from Sapodilla (Manilkara zapota) peel and seeds obtained by ultrasound-assisted technique. Research, Society and Development, 9(8), e354985158. https://doi.org/10.33448/rsd-v9i8.5158.

Asfaram, A., Ghaedi, M. M., \& Purkait, K. (2017). Novel synthesis of nanocomposite for the extraction of sildenafil citrate (Viagra) from water and urine samples: process screening and optimization. Ultrasonics Sonochemistry, 38, 463-472. https://doi.org/10.1016/j.ultsonch.2017.03.045.

Ayyanar, M., \& Subash-Babu, P. (2012). Syzygium cumini (L.) Skeels: a review of its phytochemical constituents and traditional uses. Asian Pacific Journal of Tropical Biomedicine, 2 (3), 240-246. https://doi.org/10.1016/S2221-1691(12)60050-1.

Banerjee, A., \& Dasgupta, N. B. (2005). In vitro study of antioxidant activity of Syzygium cumini fruit. Food Chemistry, 90 (4), 727-733. https://doi.org/10.1016/j.foodchem.2004.04.033. 
Barcia, M. T. (2009). Composição Centesimal e de Fitoquímicos em Jambolão (Syzygium cumini). Dissertação de Mestrado, Universidade Federal de Pelotas, RS, Brasil. Recuperado de http://guaiaca.ufpel.edu.br:8080/handle/123456789/1306.

Belwal, T., Dhyani, P., Bhatt, I. D., Rawal, R. S., \& Pande, V. (2016). Optimization extraction conditions for improving phenolic content and antioxidant activity in Berberis asiatica fruits using response surface methodology (RSM). Food Chemistry, 207, 115-124. https://doi.org/10.1016/j.foodchem.2016.03.081.

Bezerra, M. F. (2015). Polpa de jambolão (Eugenia jambolana Lam.) fresca e desidratada: características físico-químicas, bioativas e funcionais, efeitos biológicos em Caenorhabditis elegans e uso para produção de frozen yogurt caprino probiótico. Tese de Doutorado, Universidade Federal do Rio Grande do Norte, RN, Brasil. Recuperado de https://repositorio.ufrn.br/handle/123456789/20097.

Biegelmeyer, R., Andrade, J. M. M., Aboy, A. L., Apel, M. A., Dresch, R. R., Marin, R., Raseira, M. C. B., \& Henriques, A.T. (2011). Comparative analysis of the chemical composition and antioxidant activity of red (Psidium cattleianum) and yellow (Psidium cattleianum var. lucidum) strawberry guava fruit. Journal of Food Science, 76 (7), 991-996. https://doi.org/10.1111/j.1750-3841.2011.02319.x.

Borges, K. (2011). Estudo das características físico-químicas e funcionalidade de bagaços de frutas tropicais desidratadas em leito de jorro. Dissertação de Mestrado, Universidade Federal do Rio Grande do Norte, RN, Brasil. Recuperado de https://repositorio.ufrn.br/handle/123456789/15809.

Boroski, M., Visentainer, J. V., Cottica, S. M., \& Morais, D. R. (2015). Antioxidantes-Princípios e métodos analíticos. Appris Editora.

Celli, G. B., Pereira-Netto, A. B., \& Beta, T. (2011). Comparative analysis of total phenolic content, antioxidant activity, and flavonoids profile of fruits from two varieties of Brazilian cherry (Eugenia uniflora L.) throughout the fruit developmental stages. Food Research International, 44 (8), $2442-2451$. https://doi.org/10.1016/j.foodres.2010.12.036.

Cheng, V. J., Bekhit, A. E. D. A., Mcconnell, M., Mros, S., \& Zhao, J. (2012). Effect of extraction solvent, waste fraction and grape variety on the antimicrobial and antioxidant activities of extracts from wine residue from cool climate. Food Chemistry, 134 (1), 474-482. https://doi.org/10.1016/j.foodchem.2012.02.103.

Constancio, V. S. (2015). Efeito da jabuticaba (Myrciaria cauliflora), do fruto da palmeira juçara (Euterpe edulis Martius) e do jambolão (Syzygium cumini) sobre o perfil lipídico, a glicemia e a endotoxemia em camundongos submetidos à dieta de cafeteria. Dissertação de Mestrado, Universidade Federal do Espírito Santo, ES, Brasil. Recuperado de http://repositorio.ufes.br/handle/10/4931.

Corrêa, A. A. A., Alessandra, M. S. A. M., Rodrigues, W. C., Hellen, V. P. H. V., Reis, J. D., \& Saber, M. L. (2018). Potencial antimicrobiano de metabólitos de Syzygium cumini (jamelão). Revista Eletrônica Acervo Saúde, 10 (1), 2080-2086. https://doi.org/0.25248/REAS375_2018.

Costa, A. M., Soares, F. L., Roque-Specht, V. F., Barbosa-Silva, D., Lima, H. C., \& Diniz, J. D. A. S. (2012). Estabilidade de antioxidantes na farinha de araticum processada a diferentes temperaturas. In: XXII Congresso Brasileiro de Fruticultura, RS, Brasil. www.bdpa.cnptia.embrapa.br/consulta/busca?b=ad\&id=940938\&biblioteca=vazio\&busca=940938\&qFacets=940938\&sort=\&paginacao=t\&paginaAtual=1 .

Djeridane, A., Hamdi, A., Bensania, W., Cheifa, K., Lakhdari, I., \& Yousfi, M. (2013). The in vitro evaluation of antioxidative activity, $\alpha$-glucosidase and $\alpha$ amylase enzyme 54 inhibitory of natural phenolic extracts. Diabetes \& Metabolic Syndrome: Clinical Research \& Reviews, 9 (4), 324-331. https://doi.org/10.1016/j.dsx.2013.10.007.

Escriche, I., \& Juan-Borrás, M. (2018). Standardizing the analysis of phenolic profile in propolis, Food Research International, 106, 834-841. https://doi.org/10.1016/j.foodres.2018.01.055.

Frauches, N. S. (2017). Efeito de extratos de jabuticaba, jamelão e jambo sobre linhagem de adenocarcinoma de cólon humano ht-29. Dissertação de Mestrado, Universidade Federal do Rio de Janeiro, RJ, Brasil. Recuperado de http://www.repositorio-bc.unirio.br:8080/xmlui/handle/unirio/10965.

Gardusi, F., Mendes, L.G., Nogueira, G.D.R., Silva, D.I.S. \& Barrozo, M.A.S. (2014). Secagem da casca da mexerica por irradiação infravermelha e comparação dos compostos bioativos desta in natura e após a secagem. Anais do X Congresso Brasileiro de Engenharia Química, Rio de Janeiro: Blucher Chemical Engineering Proceedings, 1, 423-427.

Giusti, M., \& Wrolastad, R.E. (2001). Characterization and measurement of anthocyanins by UV-visible spectroscopy. Current Protocols in Food Analytical Chemistry, 0 (1), F1.2.1 - F1.2.13. https://doi.org/10.1002/0471142913.faf0102s00.

Gulcin, I. (2012). Antioxidant activity of food constituents: an overview. Archives of Toxicology, 86 (3), 345-391.https://doi.org/0.1007/s00204-011-0774-2.

Huang D., \& Prior, R. L. (2005). The chemistry behind antioxidant capacity assays. Journal of Agricultural and Food Chemistry, 53 (6), $1841-1856$. https://doi.org/10.1021/jf030723c.

Jing-En, L., Song-Tao, F., Zeng-Hui, Q., Chang, L., \& Shao-Ping, N. (2015). Total flavonoids content, antioxidant and antimicrobial activities of extracts from Mosla chinensis Maxim. cv. Jiangxiangru. LWT-Food Science and Technology, 64 (2), 1022-1027. https://doi.org/10.1016/j.1wt.2015.07.033.

Kaneria, M., Baravalia, Y., Vaghasiya, Y., \& Chanda, S. (2009). Determination of antibacterial and antioxidant potential of some medicinal plants from Saurashtra region, India. Indian Journal of Pharmaceutical Sciences, 71 (4), 406-412. https://doi.org/10.4103/0250-474X.57289.

Maran, J. P., Sivakumar, V., Thirugnanasambandham, K., \& Sridhar, R. (2014). Extraction, multi-response analysis, and optimization of biologically active phenolic compounds from the pulp of Indian jamun fruit. Food Science Biotechnology, 23 (1), 9-14. https://doi.org/10.1007/s10068-014-0002-y.

Martins, J. G. P. (2011). Atividade antimicrobiana de produtos naturais: erva mate e resíduos agroindustriais. Dissertação de Mestrado, Universidade de São Paulo, SP, Brasil. https://teses.usp.br/teses/disponiveis/11/11141/tde-28062011-080820/pt-br.php\#: :text=Os\%20resultados\%20demonstraram\% 20o\%20potencial,monocytogenes\%20e\%20S.

Migliato, K. F., Mello, J. C. P., Higa, O. Z., Rodas, A. C. D., Correa, M. A., Mendes, G. M. J. S., Fusco, A. A. M., Pizzolitto, A. C., \& Salgado, H. R. N. (2010). Antimicrobial and cytotoxic activity of fruit extract from Syzygium cumini Skeels. Latim American Journal of Pharmacy. 29 (5), $725-730$. 
Migliato, K. F., Corrêa, M. A., Salgado, H. R.N., Tognolli, J.O., Sacramento, L. V.S., Mello, J. C. P., Giannini, M. J. S. M., Almeida, A. M. F, \& Pizzolitto, A. C. (2011). Planejamento experimental na otimização da extração dos frutos de Syzygium cumini (L.) skeels. Química Nova, 34 (4), 695-699. https://doi.org/10.1590/S0100-40422011000400024.

Mothana, R. A. A., \& Lindequist, U. (2005). Antimicrobial activity of some medicinal plants of the island Soqotra. Journal of Ethnopharmacology, 96, 177181. https://doi.org/10.1016/j.jep.2004.09.006.

Mohamed A. A., Ali S. I., \& El-Baz, F. K. (2013). Antioxidant and antibacterial activities of crude extracts and essential oils of Syzygium cumini leaves. PLoS ONE, 8 (4) e60269. doi:10.1371/journal.pone.0060269.

Neves, B. S. S. (2017). Casca do jambolão: caracterização, planejamento e desenvolvimento de embalagens biodegradáveis ativas. Dissertação de Mestrado, Universidade Federal de Sergipe, SE, Brasil.

Oliveira, S. C., \& Andolfatto, S. (2014). Otimização do processo de extração de compostos bioativos da própolis produzida por abelhas geneticamente modificadas. Trabalho de Conclusão de Curso, Universidade Tecnológica Federal do Paraná, PR, Brasil. http://repositorio.roca.utfpr.edu.br/jspui/handle/1/2199.

Oliveira D. S. (2014). Nova metodologia para extração de compostos fenólicos de vinho tinto e avaliação da estabilidade dos extratos obtidos. Tese de Doutorado, Universidade Federal de Viçosa, MG, Brasil. https://locus.ufv.br//handle/123456789/495.

Oroian, M., Ursachi, F., \& Dranca, F. (2020). Influence of ultrasonic amplitude, temperature, time and solvent concentration on bioactive compounds extraction from propolis. Ultrasonics Sonohemistry, 64, 105021. https://doi.org/10.1016/j.ultsonch.2020.105021.

Pacheco, S. M. (2015). Frutos da família Myrtaceae: Caracterização físico-química e potencial inibitório da atividade das enzimas digestivas. Dissertação de Mestrado, Universidade Federal de Pelotas, RS, Brasil. http://guaiaca.ufpel.edu.br:8080/handle/prefix/3055.

Rai, D. R., Chadha, S., Kaur, M.P., Jaiswal, P., \& Patil, R.T. (2011). Biochemical, microbiological and physiological changes in Jamun (Syzygium cumini L.) kept for long term storage under modified atmosphere packaging. Journal of Food Science and Technology -Mysore, 48(3), 357-365. https://doi.org/10.1007/s13197-011-0254-y.

Rezende, L. C. (2010). Avaliação da atividade antioxidante e composição química de seis frutas tropicais consumidas na Bahia. Tese de pós-graduação, Universidade Federal da Bahia, BA, Brasil. Recuperado de http://www.repositorio.ufba.br/ri/handle/ri/10639.

Rufino, M. S. M., Alves, R. E., Brito, E. S., Perez-Jimenez, J., Saura-Calixto, F., \& Mancini-Filho, J. (2010). Bioactive compounds and antioxidant capacities of 18 non-traditional tropical fruits from Brazil. Food Chemistry, 121 (4), 996-1002. https://doi.org/10.1016/j.foodchem.2010.01.037.

Sanchez-Gonzalez, L., Pastor, C., Vagas, M., Chiralt, A., Gonzalez-Martinez, C., \& Chafer, M. (2011). Effect of hydroxypropylmethyl cellulose and chitosan coatings with and without bergamot essential oil on quality and safety of cold-stored grapes. Postharvest Biology and Technology, 60 (1), 57-63. https://doi.org/10.1016/j.postharvbio.2010.11.004.

Santiago, M. C. P. A., Gouvea, A. C. M. S., Peixoto, F. M., Borguini, R. G., Godoy, R. L. O., Pacheco, S., Nascimento, L. S. M., \& Nogueira, R. I. (2016). Characterization of jamelão (Syzygium cumini (L.) Skeels) fruit peel powder for use as natural colorant. Fruits, 71 (1), 3-8. https://doi.org/10.1051/fruits/2015041.

Santos, D. T., Veggi, P. C., Meireles, M., \& Angela A. (2010). Extraction of antioxidant compounds from Jabuticaba (Myrciaria cauliflora) skins: Yield, composition and economical evaluation. Journal of Food Engineering, 101 (1), 23-31. https://doi.org/10.1016/j.jfoodeng.2010.06.005.

Sari, P., Wijaya, C. H., Sajuthi, D., \& Supratman, U. (2012). Color properties, stability, and free radical scavenging activity of jambolan (Syzygium cumini) fruit anthocyanins in a beverage model system: natural and copigmented anthocyanins. Food Chemistry, 132 (4) 1908-1914. https://doi.org/10.1016/j.foodchem.2011.12.025.

Savi, A. (2015). Otimização do processo de extração de compostos bioativos de folhas de jambo (Syzygium malaccense). Trabalho de Conclusão de Curso, Universidade Tecnológica Federal do Paraná, PR, Brasil. Recuperado de https://repositorio.roca.utfpr.edu.br/jspui/handle/1/5665.

Shafi, P.M., Rosamma, M. K., Jamil, K., \& Reddy, P. S. (2002). Antibacterial activity of Syzygium cumini and Syzygium travancorium leaf essential oils. Fitoterapia, 73 (5),414-416. https://doi.org/10.1016/s0367-326x(02)00131-4.

Silva, P. B. (2014). Secagem de resíduos de frutas em secador roto-aerado. Dissertação de Mestrado, Universidade Federal de Uberlândia, MG, Brasil. https://repositorio.ufu.br/handle/123456789/15263.

Thaipong, K., Boonprakob, U., Crosby, K., Cisneroszevallos, L., \& Bryne, D.H. (2006). Comparison of ABTS, DPPH, FRAP and ORAC assays for estimating antioxidant activity from guava fruit extracts. Journal of Food Composition and Analysis, 19 (6), 669-675. https://doi.org/10.1016/j.jfca.2006.01.003.

Vizzoto, M., \& Pereira, M. C. (2008). Caracterização das propriedades funcionais do jambolão. Boletim de Pesquisa e Desenvolvimento, Embrapa, RS, Brasil. https://www.embrapa.br/busca-de-publicacoes/-/publicacao/746899/caracterizacao-das-propriedades-funcionais-do-jambolao.

Wang, L. C. \& Weller, L. (2006). Recent advances in extraction of nutraceuticals from plants. Trends in Food Science \& Technology,17 (6), 300-312. https://doi.org/10.1016/j.tifs.2005.12.004. 